\title{
A Fast Evaluation and Research Method for Exploration Potential of Oversea Basins
}

\begin{abstract}
Oversea hydrocarbon exploration always faces some problems, such as multiple basin types, different exploration prospects, various exploration degrees, diverse data quantities and geological recognitions. This paper focuses on overseas basins with different exploration degrees and offers a new evaluation system and research method for fast evaluation and risk analysis on hydrocarbon potential. Based on the theory of probability, this new evaluation system evaluates each parameter and core accumulation factor with comprehensive and quantitative assignment, to accelerate geological evaluation. In addition, this paper suggests that source rock evaluation should be the core criterion in screening evaluation of poorexplored basins, and also proposes that both the exploration potential and geological recognition should drive the evaluation for basins with high exploration degrees. This new system and method, which is an effective system for fast basin evaluation, is suggested to be used in oversea evaluation and decision-making objectively, scientifically and efficiently.
\end{abstract}

Keywords: geological risk analysis, probability model, evaluation factors, oversea exploration, hydrocarbon exploration

\section{Introduction}

Sedimentary basins are the major sites for hydrocarbon, and are the major targets of overseas hydrocarbon exploration and development. There are several challenges in oversea exploration, such as the multiplicity of basin types, the complexity of geological conditions, the different levels of data acquisition, the variety of geological recognitions and the diversity of exploration degrees.

Manuscript received May 13, 2016; accepted October 30, 2016

Hong-quan Kang ( $(\bowtie)$, Yun-hua Deng, Huai-cun Jia, Tao Cheng, Bo Bai CNOOC Research Institute, Beijing 100028, China

Email: kanghq@cnooc.com.cn
Also, it is common that the decision-makings from accumulation factors and potential studies have to be finished in a short period, because the oversea exploration projects have the attributes of short schedule and fast progress. As in published papers, commercial database and data room visit are the merely available data acquisition approaches for overseas basins hydrocarbon potential studies. As a result, the lack of data and difficulties in data acquisition significantly limit the progress of evaluation and decision-making in studies of accumulation factors and exploration potential (Cai, 2003; Guo et al., 2007; Kuang, 2007; Wang, Shi, Liu, \& Huo, 2010).

Therefore, the construction of the new system and method is of great importance, which facilitates fast hydrocarbon potential evaluation of oversea basins (Guo et al., 2007; Wang, Shi, Liu, \& Huo, 2010). It could either be the guidance for oversea hydrocarbon exploration and strategic region research, leading to earlier discoveries of potential basins or prospects, or be the technical support for oversea bidding bound.

\section{Purposes and principles for fast basin evaluation}

2.1 Purposes for basin classification and fast geological evaluation

The main purposes for overseas basin evaluation include determining the petroleum geological factors in basins of different types and exploration degrees, identifying fundamental rules and controlling factors of hydrocarbon origin, enrichment and accumulation, selecting potential prospects or layers (reservoir forming combination) and indicating long-range prospective basins (evaluation order). Finally, and comprehensively, these purposes lead to be well supportive in acquisition of high quality overseas exploration properties.

According to different exploration degrees, geological understandings and data qualities, we can classify all basins 
into three types, including basins with low exploration degree, basins with medium exploration degree and basins with high exploration degree. The basins with low exploration degree commonly indicate the frontier regions with few or without seismic surveys and drilled wells. In other cases, some wells are drilled in these regions, but only in shallow strata. The basins with medium exploration degree always have certain number of drilled wells with relatively low coverage densities. These regions may have seismic surveys, but with inadequately covered rates. The basins with high exploration degree have highly covered $2 \mathrm{D}$ or $3 \mathrm{D}$ seismic surveys and drilled wells, with certain traps inside basin drilled.

\subsection{Principles for fast geological evaluation}

According to different basin types and complex geological conditions, exploration potential and geological risks differ in variable basin types. To evaluate the exploration potential and risk differences objectively, we follow the six principles in risk evaluation of different basins with different exploration degrees (Guo et al., 2007; Kuang, 2007; Wang, Kuang, \& Zeng, 2009; Wang, Shi, Liu, \& Huo, 2010).

(1) Probability should be the essential principle in risk evaluation of overseas basins (Cai, 2003; Wang, Kuang, \& Zeng, 2009). Evaluating the geological factors in hydrocarbon accumulation means to estimate the success probabilities of core factors in hydrocarbon accumulation in basins with different exploration degrees and of different types. In addition, the probabilities of hydrocarbon accumulation qualities should be considered.

(2) The major target of geological evaluation is hydrocarbon basin. The selection of potential prospects should be carried but by evaluating probabilities and qualities of accumulation factors and by matching all these factors dynamically.

(3) The core element of geological risk evaluation should be determined according to the exploration level of target basin. It is the core element that affects the following exploration decision and the implementation of the current basin.

(4) The probability of assignment of each accumulation factor should be determined based on analogy of regional geological conditions, level of geological understanding of target basin, degree of basin exploration, and abundance of available data.

(5) The evaluation for each accumulation factor should be independent.

(6) The success probability should range from 0 to 1 . The probability 1 means the given factor absolutely exists, and the probability 0 means the given factor is absolutely absent.

\section{3 "Source rock evaluation" as the key of evaluation system and research methods in low exploration degree basins}

3.1 Rapid geological evaluation method and process for overseas low exploration degree basins

For underexplored basins, the basic idea of evaluating exploration potential is to optimize exploration direction with the core, which is source rock evaluation, and with the emphasis, which is generative hydrocarbon sags and favorable accumulation combination. The strategic region selection evaluation system and process are established with the core idea of "source rock evaluation" for new exploration region (Figure 1). Therefore, the evaluation of "five conditions" of source rocks is the key of the geological evaluation in low exploration degree basins to find out whether the basin has exploration potential or not. The "five conditions" include sedimentary and regional tectonic evolution, basin structure and generative hydrocarbon sag distribution, the geochemical indicators of source rocks and hydrocarbon discovery, well and seismic characteristics and analogy of related basins, and source rocks buried depth and maturity and hydrocarbon generation.

\subsection{Source rock evaluation model and probability assignment standard in low exploration degree basins}

In the process of source rock evaluation, to find out whether the basin has the potential for further evaluation, the "dual drive" model of source rock condition and understanding degree is adopted, with the success probability assignment of the "five conditions" of source rocks. In this evaluation model, the source rock condition is divided into 11 levels with increasing source rock quality. Better source rock condition indicates greater chance of successful drill. With the research progress on-going, the quality of source rocks would develop to good or poor quality direction. The level of understanding degree is determined by three basic parameters, including the number of data, the data quality and geological understanding, according to the principle of bucket. For example, when the data quality and geological understanding are both in the higher 5th level while the number of data being lower in the 3rd level, the final probability assignment can only be in Level 3 according to the cognition degree, the probability of comprehensive success of source rock in the basin should be 0.7 or 0.3 .

Then the classification of "five conditions" of source rocks and the assignment standard of success probability are established on this basis: higher level means higher certainty degree, higher score means better quality; the assignment standard of success probability includes 6 levels of the three basic parameters, which are "the number 


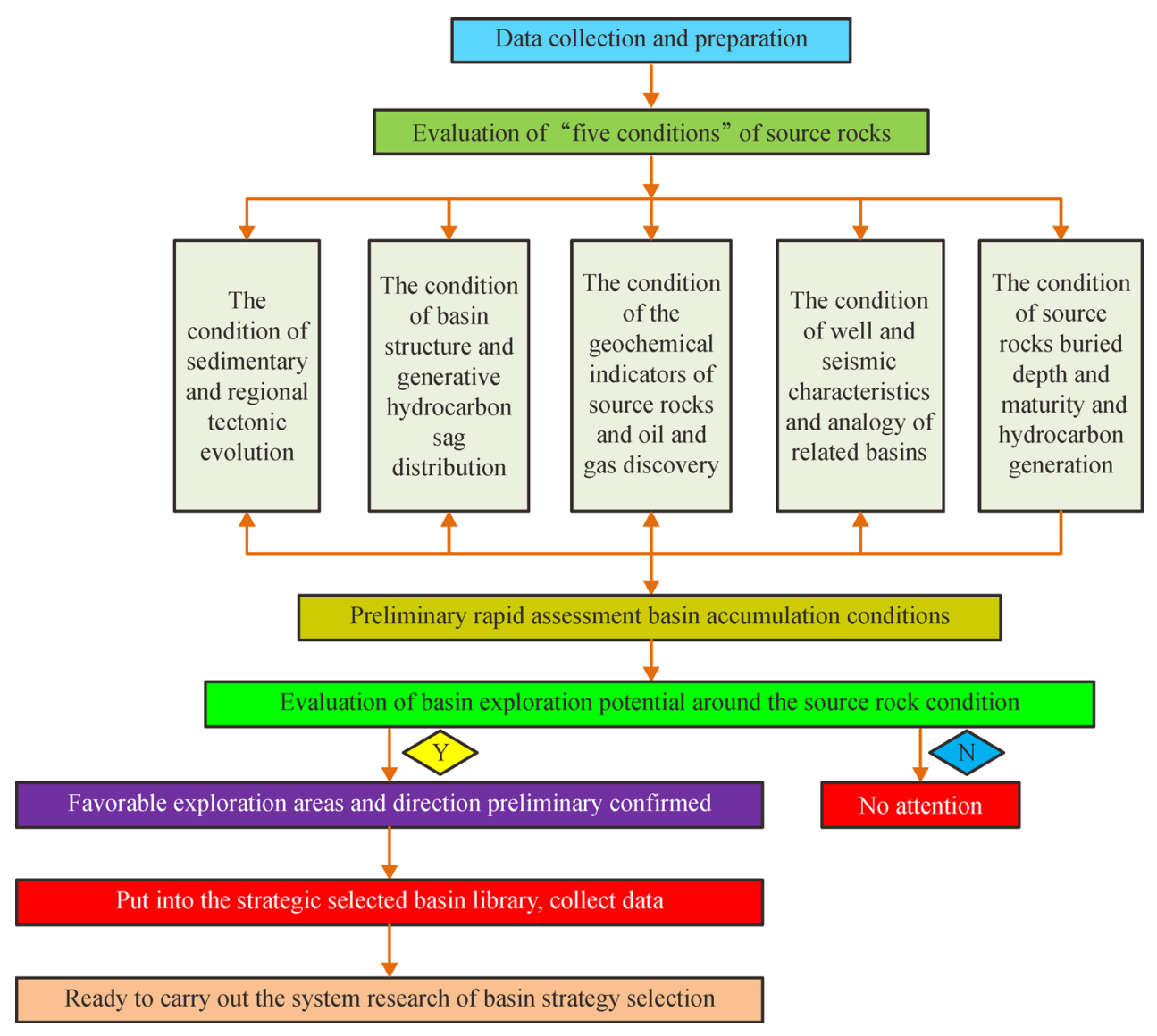

Figure 1. Evaluation method and strategic screening process in low exploration degree basins.

of data, the data quality and geological regularity", and 11 standards of "five conditions" of source rocks.

\section{4 "Accumulation conditions evaluation" as the key of evaluation system and research methods in high exploration basins}

4.1 Rapid geological evaluation method and process of overseas high exploration basins

Mature basins often have plenty of seismic and drilling data, and their petroleum geological conditions and petroleum systems have been proven. Therefore, the main part of strategic selections on overseas high exploration basins should be accumulation conditions evaluation (Figure 2), emphasizing on effective hydrocarbon generation sag evaluation and the point of the advantage direction of oil and gas, aiming at main accumulation combinations and most favorable zones, to put forward potential exploration zones and blocks for biding selection. The analysis of oil and gas accumulation conditions include 7 aspects, including analyzing sedimentary and regional tectonic evolution, basin structure and trap development, source rocks evaluation and effective hydrocarbon sag distribution, sedimentary reservoir and their distribution, hydrocarbon accumulation regularity and main control factors, cap rock and favorable accumulation combination, and predicting the favorable exploration area.

4.2 Exploration potential evaluation model and probability assignment standard in high exploration basins

Referring to the strategic basin selection evaluation method and theory "source rock evaluation as key" as reference, the "dual drive" model of exploration potential and understanding degree is established in high exploration degree basins. Based on this model, the success probability assignment of the basin exploration potential is carried out for the 7 accumulation conditions to evaluate and select the favorable zones in basins. In this evaluation model, the exploration potential is divided into 11 levels. The understanding degree of exploration potential is also divided into 6 levels according to the principle of bucket. For example, when the data quality and geological understanding are both in the higher 5th level while the number of data are lower in the 2nd level, the final probability assignment can only be in Level 2 . According to the understanding degree, the exploration potential comprehensive assignment of the basin should be 0.6 or 


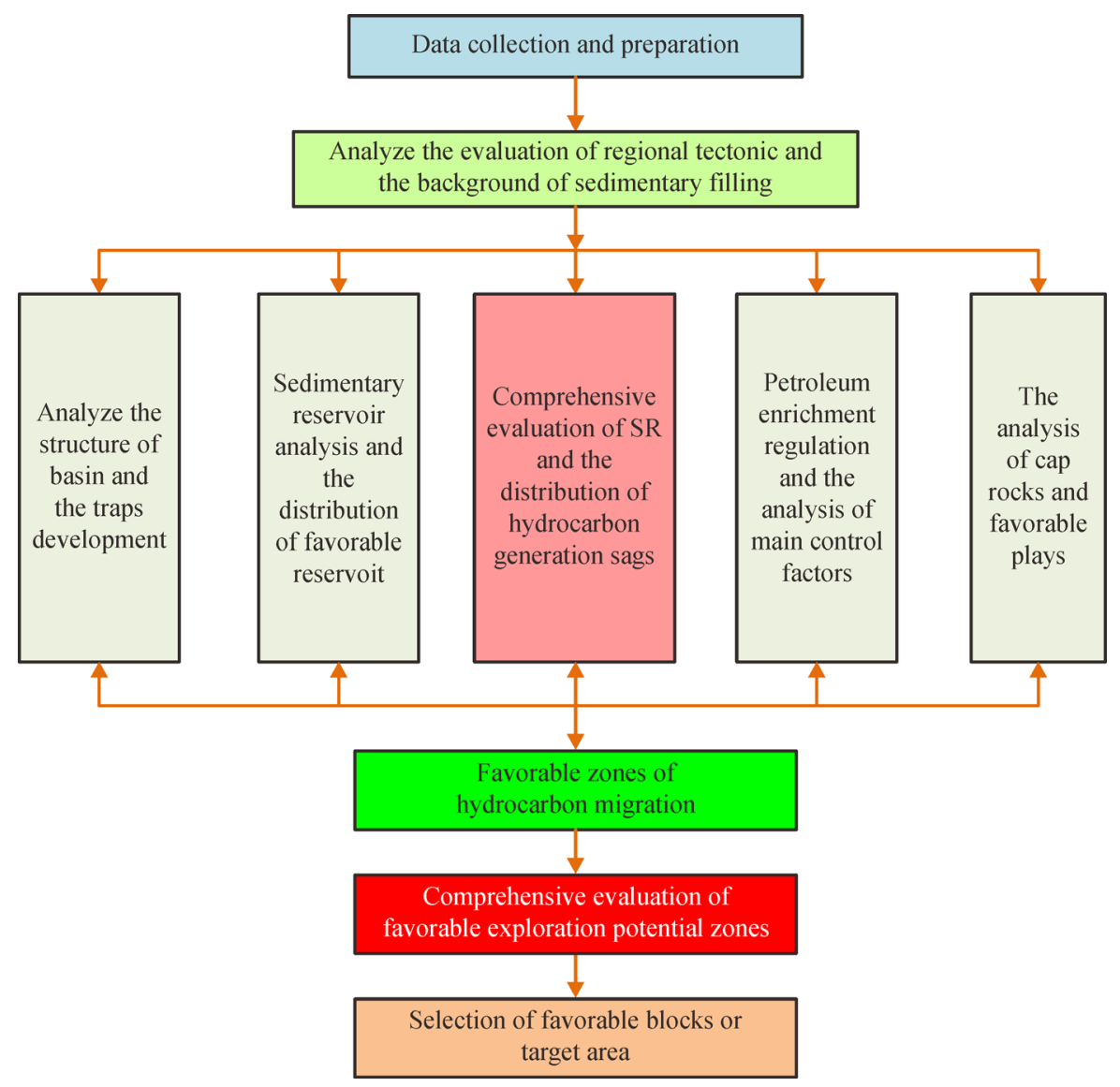

Figure 2. Evaluation method and strategic basin selection process in oversea high exploration degree basins.

\section{4 .}

Then the classification of strategic basin selection exploration potential and the assignment standard of success probability are established on this basis: Higher score means larger potential and higher success probability. The assignment standard of success probability includes 6 levels of the three basic parameters, which are "the number of data, the data quality and geological regularity" and 11 standards of basin exploration potential.

The method, process and parameter system of strategic basin selection mentioned above can be used in different stages of region selection studies. The evaluation of geological conditions understanding level (equivalent to an expert scoring system), added in the assignment standard established above, together with the "dual drive" model adopted, make the evaluation of basins and zones more scientific and objective.

\section{Applications}

Based on this regional geological evaluation system, a rapid geological evaluation of several salt basins on the Atlantic Strait has been carried out, and a number of favorable exploration basins have been optimized. Take the Santos basin of Brazil as an example, the main exploration strata are under-salt of the lower Cretaceous and the degree of exploration is relatively high with a large amount of good quality drilling and three-dimensional seismic data. Thus, the geological understanding degree is considered to beat Rank 5. Meanwhile, the tectonic pattern of "HorstGraben Structure" of under-salt rift layers has been proved by numerous drilling and seismic data, and large uplift structural belt and traps relatively develop. Drilling data indicate that the lacustrine source rock is excellent: Total organic carbon (TOC) generally at around 5\%, up to a maximum of $15 \%$. Hydrogen index (HI) mainly in more than $700 \mathrm{mg} \mathrm{HC} / \mathrm{g}$ TOC, with kerogen of Type I, hydrocarbon generation potential index $\mathrm{S} 1+\mathrm{S} 2$ is much larger than $10 \mathrm{mg} \mathrm{HC/g}$ rock. Basin modeling indicates wide distribution of effective hydrocarbon generating sag. The under-salt carbonate reservoir develops, and the overlying thick salt rock can be used as a high-quality cover layer. Furthermore, three under-salt favorable zones are identified, and the comprehensive analysis shows that the Santos basin has superior oil and gas reservoir forming condition, and the geological risk is relatively low, so the probability of geological success is up to 0.9 . Since 2006, 
in the three-under-salt favorable zone, a large number of world-class discoveries, such as Lula oilfield, Franco oilfield and Libra oil field have been obtained. Among them, Lula Oil Field has $2 \mathrm{P}$ recoverable reserves of 8.5 million tons, which proves that the Santos basin has superior reservoir condition, low geological risk and great exploration potential.

\section{Conclusions}

(1) The rapid geological evaluation and risk analysis of oversea basins' exploration potential are important parts of the oversea oil and gas exploration activities of Chinese petroleum companies.

(2) The new research method and geological evaluation system for evaluating the exploration potential of overseas basins present a set of hydrocarbon evaluation processes for the overseas basins with different exploration degrees, a quantitative assessment of the geological success probability of key hydrocarbon accumulation factors and a comprehensive judgment of exploration potential. Then the overseas basins evaluation and exploration decision can be implemented objectively, scientifically and quickly.

(3) The new research method of rapid geological evaluation of oversea basins' exploration potential is a set of effective evaluation systems. It can be used in the early stage of oversea oil and gas exploration to guarantee rapid geological evaluation and potential optimization and improve work efficiency to a great extent.

\section{References}

Cai, Q. (2003). Evaluation method and model of risks in oil-gas exploration. Techno-Economics in Petrochemicals, 27(6), 58-62 (in Chinese).

Guo, Y., Jiang, Y., Zhao, L., Li, C., Gao, L., Yang, W., Zhang, Y., \& Wu, S. (2007). Methods for petroleum exploration resource replacement strategy research in mature exploration area. Acta Petrolei Sinica, 28 (1), 20-26 (in Chinese).

Kuang, J. (2007). Geological risk assessment method of oil and gas exploration on zone and prospective trap combination. Natural Gas Industry, 27(11), 121-124 (in Chinese).

Wang, Z., Kuang, J., \& Zeng, J. (2009). Oil and gas exploration risk assessment: State and tendency of domestic and foreign researches. Scientific and Technological Management of Land and Resources, 26 (2), 125-130 (in Chinese).

Wang, J., Shi, D., Liu, Z., \& Huo, H. (2010). Discussion on method for geological risk assessment of oversea hydrocarbon exploration projects. China Petroleum Exploration, (3), 67-72 (in Chinese). 